\title{
Body Image Dissatisfaction (BID) from an Indigenous Alaska Native Female Perspective (A Pilot Study)
}

\author{
Karaline Naegele \\ University of Alaska Fairbanks \\ Christine R. Cook \\ University of Alaska Fairbanks
}

\begin{abstract}
The study was conducted as a preliminary investigation regarding body image dissatisfaction (BID) in Indigenous females living in Alaska. As BID has been a notable area of concern for European American females, and a growing concern for several other cultural groups in North America, it is important to determine whether BID is a concern for the Alaska Native population. The research was comprised of qualitative interviewing methods. Interviews were conducted with Alaska Native female participants between the ages of 18 and 23 years who were attending the University of Alaska Fairbanks. Research questions addressed whether or not Indigenous Alaska Native females experience BID, and if so how BID develops and manifests for this population. The study found that all participants experienced BID as young adults. The manifestation of BID varied on an individual basis, as seen in other research findings. Participants provided suggestions for working with Indigenous Alaskan females in regards to BID.
\end{abstract}

The Northern Review 45 (2017): 141-160 
Paul Schilder (1935) introduced the term body image as being the image or physical perception one has of his or her own body. One facet of this is body image dissatisfaction (BID)-one's dissatisfaction with his or her perceived physical body (Thompson et al., 1999; Schilder, 1935). BID is important to address as it has been linked to low self-esteem, suicidal ideation, depression, and risky behaviours (Van den Berg et al., 2010; Bearman \& Stice, 2008; Brausch \& Muehlenkamp, 2007). The term risky behaviors encompasses a variety of potentially harmful behaviors including substance or alcohol abuse, self-harm, and unprotected sexual activity (Cakar \& Tagay, 2017).

Research consistently finds high levels of BID in European American females (Cowan \& Heselmeyer, 2011; Dalley, Buunk, \& Umit, 2009; Bearman, Presnell, Martinez, \& Stice, 2006). Westernized young adult females are viewing their bodies as projects in need of constant improvement (Juarascio et al., 2011; Vartanian, 2009; Dittmar, 2007). In 2013, Michelle Obama addressed the issue of BID in the United States as part of her "Let's Move!" program, which encourages kids to get active and promotes better nutrition (Miller, 2013). Obama stated that, as a country, the United States "should really talk to kids about how they feel inside, so that we're not just dealing with the physical manifestations of the challenge" (p. 1). Acknowledging the widespread problem of negative body image in the United States, the former first lady suggested BID be addressed in a healthy and supportive way.

Yet there is a gap of BID knowledge concerning the experience of Indigenous peoples in Alaska. As BID has been a notable area of concern for European American females, and a growing concern for several other cultural groups in America, it is important to know if BID is a concern for the Alaska Native population. Furthermore, research on the Alaska Native population in particular has shown a strong prevalence of low self-esteem, depression, risky behaviour, and suicide (DeCou, Skewes, \& Lopez, 2013; Shaughnessy, Doshi, \& Jones, 2004). Additionally, according to the United States Centers for Disease Control and Prevention (2015), the U.S. suicide rates were highest in Indigenous populations both in Alaska and in the Lower 48 states. While the correlational behaviours are not the focus of this research, the established link between BID and low selfesteem, depression, and risky behaviours indicates that BID should be an area of interest for researchers and practitioners serving Alaska Native populations. 
Research concerning BID in Alaska Native populations has been both limited and outdated; thus, the purpose of this pilot study was to begin to examine BID perspectives in Alaska Native females-whether they experience BID and, if so, how it may manifest. The expectation was if Alaska Native females experience BID, their experiences and the degree of dissatisfaction would vary depending on individual differences in acculturation, self-esteem, and ethnic identity (Van den Berg et al., 2010). Understanding the experience of Indigenous peoples in Alaska is important for providing effective services. This is especially true given the correlation between BID and other mental health concerns such as risky behaviour, depression, and suicidal ideation (Van den Berg et al., 2010; Bearman \& Stice, 2008; Brausch \& Muehlenkamp, 2007).

\section{Body Image Dissatisfaction}

Body image dissatisfaction has been identified as a concern for European American female populations. Subsequently, this population has been the focus of the majority of BID studies. One of the main conclusions drawn from the research is the concept of a thin ideal; an ideal slim figure to which women compare themselves (Juarascio et al., 2011). One cause of BID and the construct of the thin ideal is exposure to media images of underweight women (Dalley et al., 2009). Constant media exposure to underweight women has aided in women's internalization that they should look underweight. BID research began as a Eurocentric issue introduced by Schidler in 1935, and it historically focused on European American female populations and thus informed therapy and counselling practices (DeLeel, Hughes, Miller, Hipwell, \& Theodore, 2009).

The literature continues to confirm that BID is a diverse issue, which permeates various races, genders, and socio-economic groups (DeLeel et al., 2009). Henrickson et al. (2010) found that African American women who identified with "White" culture, regardless of age or education, were more dissatisfied with their bodies than those who identified with African American culture. Similarly, in a study regarding Asian and Hispanic adolescents, those who reported higher levels of Western acculturation also reported higher levels of BID and depression (Xie et al., 2010). The same trends in Western influence and a rise in BID have been found within China (Holmqvist \& Frisen, 2010). These findings illustrate the concept of acculturation and its role in BID (Berry, 1997). Acculturation, defined by Berry (1997), includes a psychological, as well as a cultural, shift due to continued exposure to people of a different cultural background. 
Four studies conducted with Native Americans found high levels of BID (Kronenfeld et al., 2010; Rinderknecht \& Smith, 2002; Story et al., 1997; Story et al., 1994). Rinderknecht and Smith (2002) incorporated BID, perceptions of healthy body weight, and the prevalence of obesity in a sample of 155 Native American youth. While the authors used the term "Native American" and compared results with studies involving Alaska Natives, the group was primarily composed of American Indian youth. The authors noted that, of the Indigenous Americans studied, approximately half expressed BID and a desire to be thinner. Kronenfeld et al. (2010) also assessed BID, though in a much smaller sample of thirty-six adult Native American women. While this sample was small, and was therefore not discussed at length, Kronenfeld et al. (2010) reported levels of BID among these women similar to those of European Americans

\section{Methodology}

This research was conducted using qualitative methods of one-on-one interviewing to allow for culturally sensitive methods of delivery, a thorough understanding of experience, and minimization of harm. This method was chosen based on the recommendation of an Alaska Native psychology scholar. While there was a list of questions for the participant to look over, it was emphasized that these were only potential questions, which may or may not pertain to the individual. Interviewing allowed the participant to guide the conversation, ensuring questions were asked only when they might be relevant to the participant and thus might explore their individual experience.

Research materials were approved by the University of Alaska Fairbanks (UAF) Institutional Review Board. Demographic information was collected with the purpose of gathering the participant's age, cultural identity, and potential level of acculturation. The thirty interview questions were evaluated for any Eurocentric language or insensitivities and were crafted with a focus on health and balance, to introduce the topic in a culturally sensitive manner. Initially, the study included a set of female figure images for the participants to use to indicate a personal figure ideal and a healthy figure ideal, similar to methods utilized by Juarascio et al. (2011). However, participant feedback during the initial interview, and further consultation with an Alaska Native scholar, led to the tool being discarded. The initial participant was uncomfortable with the method and the researcher deemed the images an inappropriate tool to use with the subsequent participants. Instead, participants were asked to select celebrity figures to represent her own healthy figure ideal, meaning a figure 
that was the epitome of health, and her own personal ideal figure, meaning how she would like to look.

The population interviewed was comprised of four University of Alaska Fairbanks students who self-identified as being Alaska Native females between the ages of eighteen and twenty-three years. Participants learned about the study via word of mouth or recruitment posters. Participants were given a copy of the interview questions and a brief description of the study and nature of data collection at least twenty-four hours prior to being interviewed. They were not provided a definition of BID as the study sought participant's definition and understanding of BID. The decision to keep the description brief was an intentional effort to limit the suggestion of BID, where it might not have been applicable. Participants who accepted the methods of the study were scheduled for an interview.

Three of the four participants were over the age of 21 while one participant was 18 years old. English was the primary language for all participants, although three spoke an additional language. Three participants lived in rural parts of Alaska-a location with a population of less than 2,500 people outside of an urban place (State of Alaska Department of Labor and Workforce Development, Research, and Analysis, 2013) - although only two of the participants spent notable time in their young adulthood in a rural location. Three of the four participants lived the majority of their lives in urban settings.

Interviews were conducted individually. The camera was discretely located in a corner of the ceiling, out of view, while the microphone was located on the table between the researcher and participant. Noise-makers, which create a humming noise, were placed outside of the closed door to muffle voices and provide maximum confidentiality.

Some questions were asked of every participant while other questions were directed by previous answers from the participant. While the interviews were all guided, participant suggestions and feedback were explored. At the conclusion of the interview, each participant was asked for any suggestions when working with Alaska Native young adult females who might be experiencing BID. The rationale for asking for suggestions came from Sue and Sue's (2008) discussion of the "White solutions" that are often applied to problems across cultures. The researcher was interested in learning about any methods that were deemed suitable by the participants.

The researcher utilized hand analysis of qualitative data, as outlined by Creswell (2008). The data is primarily communicated using a "realist" 
approach, meaning an assertive presentation of fact rather than passive description (Van Maanen, 1988). This approach was chosen, in part, to minimize the researcher's re-wording and summarizing of the conversation. A realist research voice minimizes Eurocentric language from the researcher and allows the participants to convey data in their own words. All names used are pseudonyms.

\section{Results}

Participant \#1 Kelly

Kelly (pseudonym) is a 23-year-old Yup'ik and European American woman. Her mother is Yup'ik and her father European American. English is her primary language. Kelly was raised in an urban city in Alaska.

Kelly was first asked about health and balance. Her answers focused on nutrition. She discussed health in terms of eating "leafy greens" and nutritious foods. She also mentioned participating in the gathering or consumption of subsistence food sources such as moose, duck, and salmon as being part of the balance aspect. Kelly mentioned hunting and harvesting meat as being an activity that would make her more healthy and balanced. She reasoned the act of harvesting meat requires exercise, and energy spent, and the food source is also healthy. Kelly described an ideal healthy and balanced person as one who eats a healthy diet specific to their needs, does not drink or smoke excessively, and regularly engages in exercise.

Kelly defined body image as being the way she sees herself in her mind, versus what she might look like in the mirror. Kelly discussed herself as being too critical and seeing flaws that no one else likely saw. Kelly's idea of body image includes one's self-esteem and whether or not they feel good about how they look. Likewise, her idea of body image dissatisfaction is not feeling comfortable with how one looks.

Kelly communicated that her ideas of body image were crafted during her middle school years. It was also at this time, around the ages of twelve or thirteen, that Kelly began feeling self-conscious and inadequate. While many of her peers, including a good friend of hers, wanted to lose weight, Kelly stated she felt too lanky. Her body was tall and very thin, unlike the curvier women she saw in movies and on magazines. Kelly described herself as being a "late bloomer," and stated, "you see all these images and it's like I don't look like that at all." She was very insecure in middle school and also throughout high school though to a lesser extent. She stated, "in my teenage years, I was definitely worried about my weight but never to 
the point of ever doing anything about it." In short, Kelly explained how she was dissatisfied with how she measured up in comparison to women she saw in the media.

Kelly was asked to give an example of an ideal healthy figure, using any celebrity. Kelly chose Jennifer Hudson, stating that Hudson had a balanced "height to weight ratio." When Kelly was asked to select her personal figure ideal, meaning how she would look if she could, she chose Natalie Portman. During the interview, Kelly was made aware of the difference between her healthy ideal figure and her personal figure ideal and seemed surprised. She had not realized that she picked a curvy woman as being very healthy and a slim and petite woman as being her personal figure ideal.

Kelly clearly relayed that although she experienced BID in the past, she currently feels very comfortable in her skin. When asked about her level of happiness, Kelly gave a 3.5 from a Likert scale of 1 to 5 . She mentioned her happiness with herself would be higher if she did not smoke and exercised more frequently.

\section{Participant \#2 Amy}

The second interview was conducted with a 22-year-old Yup'ik female. Amy speaks some Yup'ik as well as being fluent in English. Amy was raised in a larger rural community, as well as a smaller community of less than 600 people.

Amy was asked what comes to mind when she thinks about health and balance. She took this phrase to mean both nutrition and mental balance. She did not, however, consider herself to be healthy and balanced. Amy communicated that she regularly consumes fast foods and processed foods, and rarely eats natural wholesome foods. For Amy, an ideal healthy and balanced person would abstain from alcohol, be involved in cultural activities, and uphold traditional values.

Amy's idea of body image was limited to someone who has a slim, model-like, or otherwise good figure. She did not understand body image to be applicable to everyone, and how they see themselves physically. Likewise, her definition of body image dissatisfaction differed from the Western meaning. While she understood BID to have negative connotation, Amy described BID as a condition where someone might be dependent on medication or mentally ill.

Amy was given an explanation of BID as it has been defined in the Western world and asked if she had ever experienced BID by that definition. 
Amy expressed that she has experienced BID and she attributes the onset to the trauma of losing a loved one in her youth. She communicated that she had felt a sense of guilt and depression over the loss. Amy also reported school bullying having an effect on her negative self-image. She moved to a village that spoke more Yup'ik than Amy had been accustomed to hearing. She stated that living in that location "might have been the birth of body image." She was bullied for looking different, having larger eyes in particular, and not being fluent in Yup'ik. This caused her to become self-conscious and insecure with herself.

Amy selected George Lopez as the celebrity who best represented a healthy ideal figure. She explained her choice by stating that Lopez is good at portraying emotions when they should be shown. She saw him as being healthy in his emotional expression. When she was asked to select a celebrity who would embody her personal figure ideal she chose the curvy, yet slender, Selena Quintanilla-Perez, a famous Hispanic singer.

While Amy stated that body image dissatisfaction was no longer a concern for her, she also added that she disliked her midsection. She admitted that while her dissatisfaction typically fluctuated from day to day, she found herself to be content for the most part. She stated she feels she is "fair" with her judgment, and is aware she could eat better or exercise but does not care enough to do so. Amy rated her overall level of happiness with her figure as a score of 3.3 out of 5 .

\section{Participant \#3 Brenda}

Brenda is a 23-year-old female who identifies as being primarily Iñupiaq, with some Swedish ancestry. Brenda has lived in seven different places in both urban and rural Alaska, though primarily urban locations, and her only language is English.

When Brenda was asked about health and balance, she responded that being healthy and balanced meant living a lifestyle of making nutritious choices for your body and mind. She discussed the need to eat fruit, vegetables, and whole grains while also limiting sugar and sodium intake. Brenda discussed eating good foods as a lifestyle, not a diet, in a way that promotes body and brain health. She further discussed the benefits of seal and whale oil in terms of supporting a healthy brain. Brenda described her own lifestyle as being relatively healthy.

Brenda discussed the fact that she grew up with a mother who had "severe body image issues." Brenda mentioned that as a child she witnessed her mother engaging in bulimic behavior, and this made her question her own body. Since entering college, Brenda is more comfortable 
with her appearance, yet having those previous experiences has left her feeling "on and off" about whether she thinks she is healthy.

Brenda described body image as being "the way one looks at themselves." She expanded that definition to include things like thoughts and concerns about fashion and hair styles. When asked to define body image dissatisfaction, she responded that it meant when people were unhappy with themselves and thought they could make changes in their appearances to be happier. According to Brenda, she developed this definition from media exposure and discussion with peers. While Brenda lived in many places, she did indicate that different locations contributed to different feelings on fashion or body image. When she lived in one rural area she said "nobody cared" about fashion; it was something kids considered more important when she lived in her urban home. However, she also mentioned age as being a primary factor. She lived in the rural area when she was younger, and lived in the urban area for her middle and high school years.

Brenda spoke at length about the primary source of her body image concerns, her mother. Brenda stated that many women in her family tend to gain weight around the midsection and her mother "struggles" with that genetic tendency. She feels her mother's own image issues had a very negative effect on Brenda and her sisters.

Brenda pinpointed her first moment of experiencing BID when she was eleven. She recalls grabbing her stomach and noticing that she "had like a little pudge." Brenda felt fat, ashamed, and out of shape. This event occurred during puberty, a time when the body changes, and Brenda recalls beginning to look different. Besieged by beautiful movie characters, body image discussions with peers at school, and her mother's ridicule, Brenda experienced years of feeling unattractive. While Brenda stated she has never engaged in extreme weight loss behaviours, she has wanted to in the past. According to her, she just could not make herself "starve" in order to feel better about herself.

Brenda chose the same celebrity to represent both her personal figure ideal and healthy ideal figure. Brenda's selection was Kim Kardashian. She relayed that she appreciates Kardashian's stronger body type. While she is mostly happy with herself, Brenda mentioned she "sometimes" feels insecure or uncomfortable with her body and does still feel guilty when she does not exercise. When asked about her level of happiness with her health and body image, using a Likert scale from 1 to 5, Brenda supplied a 5 . 
Participant \#4 Hannah

Hannah is an 18-year-old female. She identifies as being both MexicanAmerican and Yup'ik. She speaks some Spanish, but English is her primary language. Hannah has lived in urban settings, though she also lived in a village with a population of less than 500 people for a short period of time.

Hannah discussed health in terms of eating nutritiously and being at a healthy weight. She regarded balance as being a state of mind. She did not consider herself to be healthy and balanced, but rather "on the road to getting there." Her explanation of healthy entailed eating salad, fruit, and lean proteins. She reiterated that changes in diet and exercise are what would make her healthier. She also spoke of the mental hurdles she was actively working to overcome in accepting herself.

Hannah defined body image as being "how you see yourself in the mirror." Similarly, she explained her idea of BID as when someone "is unhappy with the way they look." Hannah also discussed the need for some level of acceptance for one's body. Hannah stated that these ideas of body image and BID came from discussions at home. Hannah's mother was often ridiculed for her weight, by Hannah's grandmother, during her youth. Hannah's mother was open with Hannah about her desire to "break the cycle" and instill a positive body image in her daughter.

Hannah first experienced BID at the age of 12 when she went from wearing a size 16 up to a size 18. The change in size alarmed her. Additionally, Hannah described other females in her middle school as being "extremely skinny." Hannah described herself as, "bigger boned ... more overweight than other girls [her] age." She relayed feeling large, tall, and not proud of herself. Other girls at the charter school she attended often gave her looks that indicated their disapproval. Hannah relayed feeling a sense of low self-esteem and depression. She ended up leaving her middle school due to harassment over her weight, acne, and her mixed racial background.

When Hannah was asked to choose a celebrity that embodied a healthy ideal figure she chose extremely thin actress Angelina Jolie. Hannah explained her choice was not just "because [Jolie's] supposed to be the most beautiful woman in the world." Hannah found Jolie's humanitarian efforts to be an inspiring role model for being healthy and balanced. However, Hannah chose a personal figure ideal to be Wonder Woman. She acknowledged Wonder Woman's active role in fighting for good and praised her Amazon-like muscular physique. Hannah added, with a smile, "I kind of relate to her because I'm big boned and I'm tall." 
Hannah remains concerned about her weight, though she regards it as primarily a health issue rather than an aesthetic one. Hannah explained she has a high risk of diabetes from both sides of her family. While she has engaged in extreme, restrictive, diet behaviours in the past, Hannah currently practices dietary moderation and exercise. On a Likert scale of 1 to 5 Hannah rated her level of happiness with her figure a 4 . She explained that while she is not completely satisfied, and experiences moments of low self-esteem and discomfort over her appearance, she is on the road to being in what she labelled as "the healthy zone."

\section{Discussion}

Common themes found in all interviews included presence of BID, age of onset, peer and media influence, fear of judgment or ridicule, selfpressures and expectations, discomfort, and emphasis on nutrition. Other themes present, though not in every interview include: importance of exercise, importance of traditional Indigenous food sources, feelings of guilt, and discrepancy between healthy ideal figure and personal figure ideal. Each participant experienced BID during the same age-range, between twelve and sixteen years old. Despite being from different areas of Alaska, both rural and urban, each participant experienced BID in either middle school or high school. All participants mentioned the influence of peers, media images, or movies. Participants described feeling like they were being judged or that they did not look how they were expected to look. The idea of an audience who was constantly assessing and judging them was a reoccurring theme in the interviews. Lastly, self-pressure and expectations were part of this phenomenon. Each participant noted their concerns, criticisms, and judgments were often self-inflicted. Their comments indicated that each of them suffered from low self-esteem at the onset of adolescence, a finding that parallels other studies (Van den Berg et al., 2010).

The literature acknowledges that Indigenous peoples, including individuals who identify as American Indian and Alaska Native, often think of life in terms of harmony and/or balance (Bassett, Tsosie, \& Nannauck, 2012; Herring, 1997). In attempting to approach interviews in a less Eurocentric way, participants were initially asked about health and balance. This phrase was chosen to allow room for cultural interpretation. To some extent, each participant gave both Western answers and as well alluded to more traditional Alaska Native views. In terms of what constituted healthy, nutrition, eating leafy greens, less consumption of fast 
food or hydrogenated oils, and consuming fruits and vegetables, were all suggestions. Most participants also discussed exercise as an important aspect of health, but participants tended to lend more non-Western ideas to the phrase balance. The word balance tended to invoke ideas about subsistence living and eating natural food sources, in order to find some sense of moderation.

Subsistence foods were a common theme amongst the interviewees. The discussion focused on nutritional food choices and sources when discussing body image and health. Participants all mentioned a desire to have healthier eating habits and discussed traditional food sources as being a primary part of eating healthier. Most participants also discussed eating fast food as a negative habit. Amy stated she often ate unhealthy foods instead of traditional Yup'ik foods. This idea of non-Indigenous cultural food as being a source for unhealthiness was one that surfaced in three of the four interviews. This is similar to findings by DeCou et al. (2013), wherein participants indicated traditional foods and practices were a contributing factor to living healthy. Unhealthy eating was correlated with the divergence from traditional food sources. Kelly expressed the desire to maintain health and balance by hunting, harvesting, and consuming moose, duck, and salmon. Amy also mentioned that she would be healthier had she, "not [been] introduced to fast foods...", as "it's not our tradition we have." Lastly, Brenda spoke extensively about the need for traditional foods, such as seal oil or whale fat, to be discussed in terms of being healthy sources of fat and an important factor in maintaining brain health. Participants, without prompt, related the idea of eating healthier or being healthier with consuming more traditional foods and limiting fast food consumption.

Every participant in this study defined BID as something negative. Participants recognized body image dissatisfaction as being a negative state of mind, or feeling you needed to look a certain way. The fact that most participants gave very Eurocentric definitions of BID indicates a certain level of acculturation. And regardless of the definitions provided, the idea of disliking one's body was not a foreign concept to any participant. Sue and Sue (2008) discuss the varying levels of acculturation that influence the extent to which an individual struggles with identity. Amy, who is from a rural location, described BID as a mental illness or a drug dependency while Hannah, Brenda, and Kelly gave answers in sync with Paul Schilder's (1935) definition. Amy's ideas of body image and BID suggest a lack of exposure to these two terms. Kelly, who is not from a rural area, communicated that her experience would likely differ 
from someone who had lived in a village. Kelly acknowledged that she felt very connected with the Western side of her culture. The participants who had primarily lived in urban Alaska articulated a more Westernized experience of BID than Amy, the participant who had primarily lived in rural Alaska. This might indicate support for a correlation between acculturation and both the prevalence and extent of BID experienced, as found in other studies (Henrickson et al., 2010; Holmqvist and Frisen, 2010; Xie et al., 2010; Berry, 1997).

Research shows that BID is commonly found amongst young adult females (Van den Berg et al., 2010; DeLeel et al., 2009). The interviews were designed to examine whether Alaska Native females might be included in that population. How does BID develop and manifest for these women? While it cannot be stated that BID develops or manifests in one specific way, the data indicates BID develops during young adulthood for some Alaska Native females in Alaska. The interviews, while clearly not providing a large enough sample to make generalizations, indicate that some Alaska Native females experienced BID during their teenage years. The "imaginary audience," as Kelly described, borrowing a term crafted by Elkind (1967), was a reoccurring theme in each interview. The imaginary audience refers to adolescent egocentrism, when adolescents believe that those around them are obsessively observing them and are concerned with their affairs (Elkind \& Bowen, 1979). All four participants experienced discomfort beginning in their adolescent years, a common finding in BID studies (DeLeel et al., 2009). Hannah noted girls in her middle school were very thin and gave her judging looks for her heavy weight. While one of the common discomforts of females in the Western world is the concern with being overweight, other discomforts can contribute to BID; Kelly worried she was too lanky and Amy experienced ridicule for her "big eyes," and Hannah also felt self-conscious about her acne. For a variety of reasons, participants described a sense of low selfesteem and discussed themselves as feeling inferior to standards either real or imagined. Similar findings by Van den Berg et al. (2010) reported a correlation between low self-esteem, depression, and BID. Furthermore, the interviews illustrate that BID can develop in women due to or in association with parental modelling behaviours, trauma, feelings of low self-esteem, depression, or the pressure of media role models. This further reinforces the connection between BID and depression, low self-esteem, and other risky behaviours. And, as stated previously, there has been research regarding the high prevalence of depression, low self-esteem, suicide, and other risky behaviours in Indigenous populations in Alaska 
(CDC, 2015; DeCou et al., 2013; Shaughnessy et al., 2004). The implications of these connections suggest that BID should be an area of research interest.

Participants gave varied answers for their ideals, but the commonality was that each individual gave a body type, for both healthy ideal figure and personal figure ideal, which they stated was not like their own body. This in itself indicates a level of dissatisfaction. For a healthy ideal figure, Kelly and Brenda chose strong and curvy women, Amy focused on mental health, and Hannah chose a very thin ideal figure while also emphasizing the humanitarian efforts of her role model. The choices made by each participant lend insight as to what they consider health to consist of and what healthy looks like to them. In many ways, their choices reinforced the definitions of health each participant gave at the start of the interview. Likewise, while most participants rated their levels of happiness as being high and concluded body image was not presently a concern, three of the four participants gave comments indicating a current level of dissatisfaction and low self-esteem. Hannah stated that she could not "go into a surgery room and say cut my bones to make them smaller. I'll always be bigger, I'll always be taller, but I can be healthier." Hannah displayed the phenomenon Hoskins (2002) found with girls seeing their bodies as projects to be improved. She indicated her dissatisfaction with not only her weight but her physical bone structure and height. Additionally, Amy expressed discomfort with the appearance of her midsection and Brenda mentioned feelings of guilt over missing workouts. The participants' experiences provided enough information to indicate that some Indigenous women in Alaska experience BID.

As previously stated, each participant was asked to provide suggestions for professionals who may work with Alaska Native young adult females experiencing BID. All of the participants suggested that practitioners and other professionals embed Alaska Native culture and traditions into the interaction; this was also found by DeCou et al. (2013). Kelly suggested learning to speak a few words of the Alaska Native person's language. Hannah's suggestions for working with Alaska Native females focused on creating a communal feeling. She suggested hosting a potlatch, featuring traditional and healthy food options, as an introduction to a group addressing body image. Hannah discussed the importance of creating a welcoming atmosphere and encouraging a feeling of community. Additionally, she noted the presence of good traditional foods, such as whale, would likely make the process more comfortable for Alaska Native females. Amy also suggested incorporating some cultural 
aspects to help make people feel comfortable. She relayed that finding out their favorite cultural activity could be helpful. She also mentioned that someone working with the Alaska Native female population might explain their motive behind wanting to understand the Alaska Native person and their culture.

Brenda's comments also suggested a need to embed culture. However, taking it a step further, her comments strongly corroborated Sue and Sue's criticism (2008) of canned Eurocentric curricula and interventions. Brenda acknowledged that sometimes BID problems can stem from inadequate knowledge of nutrition. Information taught in schools did not translate to her living situation. She verbally painted a picture of living in a community of less than 200 people, on the western coast of Alaska. She described the lone grocery store of the village, one shelf full of soda pop, a refrigerated section full of pre-made pizzas and void of eggs, milk, fruit, and vegetables. While she learned she should eat fruits, whole grains, vegetables, and milk, few, if any, of these items could ever be found in her village. School curriculum also taught her that fats were bad. Brenda questioned her traditional food sources of whale fat, and seal and fish oil. She communicated that, because the information in school conflicted with her traditional food sources, she did not know what she was supposed to eat. Brenda stated that the poorly executed nutrition information "contributes to weight and our choices ... when we come to the city we don't know what these foods are so we fall back to our comfort zone and grab a soda instead of a pomegranate juice ... because I don't know what [pomegranate juice] is." While Brenda, herself, clearly knew about pomegranate juice, she illustrated an issue that faces many Alaska Natives who leave the village to live in more urban locations. Brenda suggested that people who work with Alaska Natives addressing nutrition and BID use information that applies to Alaska Natives. She indicated that it is not helpful to continue to hammer in the knowledge that one should eat eggs or fruit if there is never any available in the village.

The study confirmed BID was experienced, provided examples of how it was experienced, and supplied suggestions for professionals working with Alaska Native females. While there is no general conclusion to pull from the participants' answers and to broadly apply to Alaska Native women as a whole, the responses provide valuable insights into their perspectives on BID and how professionals might establish rapport for intervention. Furthermore, given correlation of BID and risky behaviours, the presence of BID in some Alaska Native females suggests a need for further research. 


\section{Limitations}

Limitations to the study are primarily due to the small sample size. The sample of four women were all students between the ages of eighteen and twenty-three. The likelihood of acculturation is higher as the females interviewed are part of a university system. To get a better sample, women who are currently living in rural Alaska, as well as those living in cities, would need to be recruited. A larger sample size would be helpful for comparing and contrasting the two groups. Lastly, there is certainly a limitation with grouping Alaska Natives into any sort of generality. For example, Athabascan (Dena) peoples are physically and culturally very different from Inuit (including Yu'pik and Iñupiaq peoples) (Langdon, 2002). Therefore, if an Athabascan individual experiences BID, this might be very different from how an Inuit person may experience BID. To address this issue, each interview has been discussed as a case study, rather than grouping participant answers. Additionally, the interviews indicated that while we can conclude BID is a potential problem, there are a variety of factors impacting exactly how it manifests for each individual.

\section{Conclusion}

The findings from these interviews support other research concerning BID in young adult females. Participants did indicate low self-esteem in adolescence and internalization of thin ideals, as found in other studies (Cowan \& Heselmeyer, 2011; Juarascio et al., 2011; Van den Berg et al., 2010; Bearman \& Stice, 2008; Crow, Eisenberg, Story, \& Neumark-Sztainer, 2008). Also, one participant mentioned that living in a rural village lent to less concern with looks or body image, similar to Holmqvist and Frisen's (2010) study on affluence and body image. However, the participant did acknowledge that the reduced concern could have been the result of her age at the time, rather than the location she was living.

While not able to be generalized, the data provide some answers to the basic questions of how BID is defined, whether it occurs, and how it develops and manifests for Alaska Native females. No generic summation of results can be applied, due to the varying nature of the interview answers. However, BID was defined as being a negative sense of body image, BID did occur for each participant, and BID manifested in ways that have been found in other studies. While the study cannot conclude BID is a "problem" for this population, it is clear BID can occur for the Alaska Native female population. Though the sample size was very small, 
there was also evidence to suggest a possible correlation between levels of acculturation and the prevalence and extent of BID, as seen in other studies (Xie et al., 2010). The three participants who had primarily lived in urban areas communicated higher levels of BID than the participant who had primarily lived in a rural village. Therefore, BID and how it interplays with levels acculturation in Alaska Native females may be another area for future research.

Furthermore, there are a few suggestions to be taken from the interviews conducted, concerning working with young adult Alaska Native females. Participants indicated that taking time to appreciate and acknowledge Alaska Native culture could greatly assist when establishing rapport. Additionally, participants indicated that traditional practices and food sources helped them to lead healthier lives, which is consistent with other research findings (DeCou et al., 2013). Also, as Sue and Sue (2008) suggested, curriculum should not be standard or Eurocentric. When working with Indigenous Alaskan populations, professionals should include language, terms, locations, foods, and events or situations that pertain to Indigenous experiences. Using standardized curriculum not only alienates the Alaska Native population but, as Brenda communicated, can confuse individuals. These suggestions, while meant for helping professionals working with Alaska Native females experiencing BID, might also be utilized by researchers studying these women's experiences.

Additional suggestions for future BID research would primarily consist of obtaining a larger and more diverse sample size. Both rural and urban females should be interviewed in future research as well as individuals outside of the university setting. Additionally, given that the health and balance responses from participants tended to refer back to cultural practices and ideas, researchers might benefit from speaking with Alaska Native elders regarding body image.

\section{Authors}

Karaline Naegele completed her M.Ed. at the University of Alaska Fairbanks and is beginning her doctoral studies in educational psychology at the University of Wisconsin Milwaukee.

Christine R. Cook is a school counsellor for the Fairbanks North Star Borough School District. 


\section{References}

Banitt, A., Kaur, H., Pulvers, K., Nollen, N., Ireland, M., \& Fitzgibbon, M. (2008). BMI percentiles and body image discrepancy in Black and White adolescents. Obesity, 16, 987-991.

Bassett, D., Tsosie, U., Nannauck, S. (2012). Our culture is medicine": Perspectives of native healers on posttrauma recovery among American Indian and Alaska Native patients. The Permanente Journal, 16, 19-27.

Bearman, S., Presnell, K., Martinez, E., \& Stice, E. (2006). The skinny on body dissatisfaction: A longitudinal study of adolescent girls and boys. Journal of Youth and Adolescence, 35, 229-241.

Bearman, S., \& Stice, E. (2008). Testing a gender additive model: The role of body image in adolescent depression. Journal of Abnormal Child Psychology, 36, 1251-1263.

Berry, J. (1997). Immigration, acculturation, and adaptation. Applied Psychology, 46 (1), 5-34.

Brausch, A., \& Muehlenkamp, J. (2007). Body image and suicidal ideation in adolescents. Body Image, 4, 207-212.

Cakar, F., \& Tagay, O. (2017). The mediating role of self-esteem: The effects of social support and subjective well-being on adolescents' risky behaviors. Education Sciences: Theory \& Practice, 17, 859-876.

Centers for Disease Control and Prevention. (2015). Racial and Gender Disparities in Suicide Among Young Adults Aged 18-24: United States, 2009-2013. Retrieved from: https://www.cdc.gov/nchs/data/hestat/suicide/racial_and_ gender_2009_2013.htm

Cowan, E., \& Heselmeyer, R. (2011). Bulimia and dissociation: A developmental perspective. Journal of Mental Health and Counseling, 33, 128-143.

Creswell, J. (2008). Educational research: Planning, conducting, and evaluating quantitative and qualitative research ( $3^{\text {rd }}$ ed.). Columbus: Pearson Education Ltd.

Crow, S., Eisenberg, M., Story, M., \& Neumark-Sztainer, D. (2008). Are body dissatisfaction, eating disturbance, and body mass index predictors of suicidal behavior in adolescents? A longitudinal study. Journal of Counseling and Clinical Psychology, 76, 887-892.

Dalley, S., Buunk, A., \& Umit, T. (2009). Female body dissatisfaction after exposure to overweight and thin media images: The role of body mass index and neuroticism. Personality and Individual Differences, 47, 47-51.

DeCou, C., Skewes, M., Lopez, E. (2013). Traditional living and cultural ways as protective factors against suicide: Perceptions of Alaska Native university students. International Journal of Circumpolar Health, 72, 142-146.

DeLeel, M., Hughes, T., Miller, J., Hipwell, A., \& Theodore, L. (2009). Prevalence of eating disturbance and body image dissatisfaction in young girls: An examination of the variance across racial and socioeconomic groups. Psychology in the Schools, 46, 767-775. 
Dittmar, H. (2007). The costs of consumer culture and the "cage within": The impact of the material "good life" and "body perfect" ideals on individuals' identity and well-being. Psychological Inquiry, 18, 23-31.

Elkind, D. (1967). Egocentrism in adolescence. Child Development, 38, 1025-1034.

Elkind, D., \& Bowen, R. (1979). Imaginary audience behavior in children and adolescents. Developmental Psychology, 15, 38-44.

Henrickson, H., Crowther, J., \& Harrington, E. (2010). Ethnic identity and maladaptive eating: Expectancies about eating and thinness in African American women. Cultural Diversity and Ethnic Minority Psychology, 16, 87-93.

Herring, R. (1997). Counseling diverse ethnic youth: Synergetic strategies and interventions for school counselors. Orlando: Harcourt Brace \& Co.

Holmqvist, K., \& Frisen, A. (2010). Body dissatisfaction across cultures: Findings and research problems. European Eating Disorders Review, 13, 133-146.

Hoskins, M. (2002). Girls' identity dilemmas: Spaces defined by definitions of worth. Health Care for Women International, 23, 231-247.

Juarascio, A., Forman, E., Timko, C., Herbert, J., Butryn, M., \& Lowe, M. (2011). Implicit internalization of the thin ideal as a predictor of increases in weight, body dissatisfaction, and disordered eating. Eating Behaviors, 12, 207-213.

Kronenfeld, L., Reba-Harrelson, L., Von Holle, A., Reyes, M., \& Bulik, C. (2010). Ethnic and racial differences in body size perception and satisfaction. Body Image, 7, 131-136.

Langdon, S. (2002). The native people of Alaska: Traditional living in a northern land ( $^{\text {th }}$ ed.). Anchorage, AK: Greatland Graphics.

Miller, T. (2013, March 4). First lady Michelle Obama talks Let's Move!, childhood body image and chubby pets in first Google+ hangout. New York Daily News. Retrieved from http://www.nydailynews.com/life-style/health/lady-michelleobama-talks-childhood-body-image-google-hangout-article-1.1278920.

Rinderknecht, K., \& Smith, C. (2002). Body-image perceptions among urban Native American youth. Obesity Research, 10, 315-327.

Schilder, P. (1935). The image and appearance of the human body. Oxford, England: Oxford University Press.

Shaughnessy, L., Doshi, S., Jones, S.E. (2004). Attempted suicide and associated health risk behaviors among Native American high school students. Journal of School Health, 74, 177-182.

State of Alaska Department of Labor and Workforce Development, Research, and Analysis. (2013, February). Urban and rural: Classification for Alaska. Retrieved from http://laborstats.alaska.gov/census/urbrur.htm.

Story, M., Hauck, F., Broussard, B., White, L., Resnick, M., \& Blum, R. (1994). Weight perceptions and weight control practices in American Indian and Alaska Native adolescents: A national survey. Archives of Pediatric and Adolescent Medicine, 148, 567-571. 
Story, M., French, S., Neumark-Sztainer, D., Downs, B., Resnick, M., \& Blum, R. (1997). Psychosocial and behavioral correlates of dieting and purging in Native American adolescents. Pediatrics, 99, 1-8.

Sue, D.W., \& Sue, D. (2008). Counseling the culturally diverse: Theory and practice ( $^{\text {th }}$ ed.). New York, NY: John Wiley \& Sons, Inc.

Thompson, J.K., Heinberg, L.J., Altabe, M., Tantleff-Dunn, S. (1999). Exacting Beauty: Theory, assessment, and treatment of body image disturbance. Washington, DC: American Psychological Association.

Van den Berg, P., Mond, J., Eisenberg, M., Ackard, D., \& Neumark-Sztainer, D. (2010). The link between body image dissatisfaction and self-esteem in adolescents: Similarities across gender, age, weight status, race/ethnicity, and socioeconomic status. Journal of Adolescent Health, 47, 290-296.

Van Maanen, J. (1988). Tales of the field: On writing ethnography. Chicago, IL: University of Chicago Press.

Vartanian, L. (2009). When the body defines the self: Self-concept clarity, internalization, and body image. Journal of Social and Clinical Psychology, 28, 94-126.

Xie, B., Unger, J., Gallaher, P., Anderson Johnson, C., Wu, Q., Chou, C. (2010). Overweight, body image, and depression in Asian and Hispanic adolescents. American Journal of Health and Behavior, 34, 476-488. 